This item was submitted to Loughborough's Research Repository by the author.

Items in Figshare are protected by copyright, with all rights reserved, unless otherwise indicated.

\title{
Review of state-of-the-art wireless technologies and applications in smart cities
}

PLEASE CITE THE PUBLISHED VERSION

https://doi.org/10.1109/IECON.2017.8217074

PUBLISHER

(C) Institute of Electrical and Electronics Engineers (IEEE)

VERSION

AM (Accepted Manuscript)

LICENCE

CC BY-NC-ND 4.0

REPOSITORY RECORD

Zhu, Hongxu, Anna S.F. Chang, Roy S. Kalawsky, Kim F. Tsang, Gerhard Petrus Hancke, Lucia Lo Bello, and Wing Kuen Ling. 2019. "Review of State-of-the-art Wireless Technologies and Applications in Smart Cities". figshare. https://hdl.handle.net/2134/28042. 


\title{
Review of State-of-the-Art Wireless Technologies and Applications in Smart Cities
}

\author{
Hongxu Zhu' ${ }^{1}$, Anna S. F. Chang ${ }^{2}$, Roy S. Kalawsky², Kim Fung Tsang ${ }^{1}$, Gerhard Petrus Hancke², Lucia Lo Bello , \\ Wing Kuen Ling ${ }^{5}$ \\ ${ }^{1}$ Department of Electronic Engineering, City University of Hong Kong, Hong Kong \\ ${ }^{2}$ Wolfson School of Mechanical, Electrical \& Manufacturing Engineering, Loughborough University, Leicestershire, U.K \\ ${ }^{3}$ Department of Computer Science, City University of Hong Kong, Hong Kong \\ ${ }^{4}$ Department of Electrical, Electronic and Computer Engineering, University of Catania, Italy \\ ${ }^{5}$ Faculty of Information Engineering, Guangdong University of Technology, China
}

\begin{abstract}
There are increasing preferences to employ wireless communication technologies for high mobility, high scalability and low-cost applications in smart city development. This paper gives a brief synopsis of typical wireless technologies in smart city applications and the comparison analysis between them. The trend for smart city wireless technology is also presented. Examples, for several key applications within smart city development (healthcare, smart grid, localization) are studied and current advanced solutions supporting these applications are summarized with futuristic trends and demands are presented.
\end{abstract}

\section{INTRODUCTION}

There are increasing preferences in industries to employ wireless communications for high mobility, high scalability and low cost smart city applications. It is estimated that the population of smart city wireless devices will reach seven trillion by 2020 and this is equivalent to one thousand devices per capita [1].

In this paper, present and futuristic state-of-the art wireless technologies are reviewed and comparison analysis is applied. For better illustration, smart city applications are conducted as case studies. Findings from advanced literature research and case study analysis will provide useful futuristic developments for researchers' reference.

The paper is organized as follows: Section II is the review of the state-of-the-art smart city wireless technologies and comparison analysis. Section III highlights some important applications applied in smart city development. Current advanced snapshots are captured. Futuristic potential wireless technologies on these applications are also studied.

\section{REVIEW OF WIRELESS STATE-OF-THE-ART WIRELESS STANDARDS}

Characteristics of wireless technologies are studied in order to provide a better understanding of the performance for WSN applications. The following advanced smart city wireless technologies WiFi, BLE, ZigBee and Lora are discussed in detail.

\section{A. WiFi}

WiFi is a popular wireless technology based on the IEEE $802.11 \mathrm{ga} / \mathrm{n} / \mathrm{ac}$. It is widely used in mobile phones, smart home, automation and networking. It performs similar to the Ethernet network but no cabling or wiring is needed. WiFi normally operates in the $2.4 \mathrm{GHz}$ or $5 \mathrm{GHz}$ frequency band which is license free worldwide. Compared with Zigbee, the major advantages of WiFi are its fast speed and high flexibility. The maximum data rate can reach $150 \mathrm{Mb} / \mathrm{s}$, which means $\mathrm{WiFi}$ has the potential to be applied in large scale network for resources sharing, data aggregation, and even big data transmission.

In contrast, there are still some challenges in using WiFi in certain smart city applications. WiFi consumes high power to facilitate the high data rate transmission. As a result, WiFi devices require $\mathrm{AC}$ power supply or else batteries must be recharged within hours, which causes inconvenience to users. Furthermore, WiFi uses star topology-hence, it suffers from single point failure. When an access point is unavailable, all other devices connected to that access point will be disconnected. The installation of WiFi is also expensive.

\section{B. $B L E$}

Bluetooth is a short-ranged ratio link for wireless transmission between mobile users. Bluetooth (Bluetooth LE, BLE, marketed as Bluetooth Smart) is a low energy wireless personal area network technology designed and marketed by the Bluetooth Special Interest Group. According to its characteristics - low power consumption, low cost and high mobility, Bluetooth can achieve good performance on many smart city applications such as healthcare, localization, etc. Compared to Classic Bluetooth, Bluetooth Smart is intended to provide considerably reduced power consumption and cost while maintaining a similar communication range. Recent improvement of Bluetooth as a real-time protocol, enhanced its importance in industrial, healthcare and other smart city applications [2]. Recently, the Mesh BLE specification was published, which provides mobility support and a short startup phase (as there is no need for establishing a connection), but it does not guarantee an upper bound on the message latency [3]. 


\section{ZigBee}

ZigBee is a low-cost, low-power standard targeted at applications that require short-range, low-rate wireless data transfer, such as wireless light switches/bulbs, smart meters, traffic management systems, etc. ZigBee is intended to be simpler and less expensive than many other wireless personal area networks. For instance, the results of the experimental assessments in [30] show that the IEEE 802.15.4/ZigBee network is able to support smaller duty cycles than 6LowPAN. According to the network topology, ZigBee has a native support of wireless mesh networking, where a ZigBee device can transmit data over a multi-hop path by passing through a mesh network of intermediate devices. This allows more devices to be connected over a wider distance.

\section{LoRa}

LoRa, a new merging wireless protocol for long distance communication, is suitable for extensive smart city applications for data aggregation, and remote data transfer (indoor/outdoor). It is invigilated that $\sim 14 \mathrm{~km}$ coverage may be achieved by LoRa [4]. Leveraged on the excellent sensitivity of LoRa, it may be a potential backbone wireless technology for inter-connection between two networks in large scale.

\section{E. IEEE 802.11p}

The advent of vehicle to vehicle (V2V) and vehicle 2 Infrastructure (V2X) within the transport sector has led to IEEE 802.11p [5] being developed which is an approved amendment to the IEEE 802.11 standard. This has been developed to support wireless access in vehicular environments. This includes data exchange between high-speed vehicles and between the vehicles and the roadside infrastructure, so called V2X communication, in the licensed ITS band of $5.9 \mathrm{GHz}$ (5.85-5.925 GHz).

\section{III. .APPLiCATIONS AND CASE STUdies OF STATE-OF-THE-ART WIRELESS TECHNOLOGIES}

\section{A. Healthcare}

World health statistical report written by World Health Organization (WHO) has raised the concern on medical support staff shortages and the aging population growth. The gap is most acute in the U.S. and U.K. for shortage of nurses.

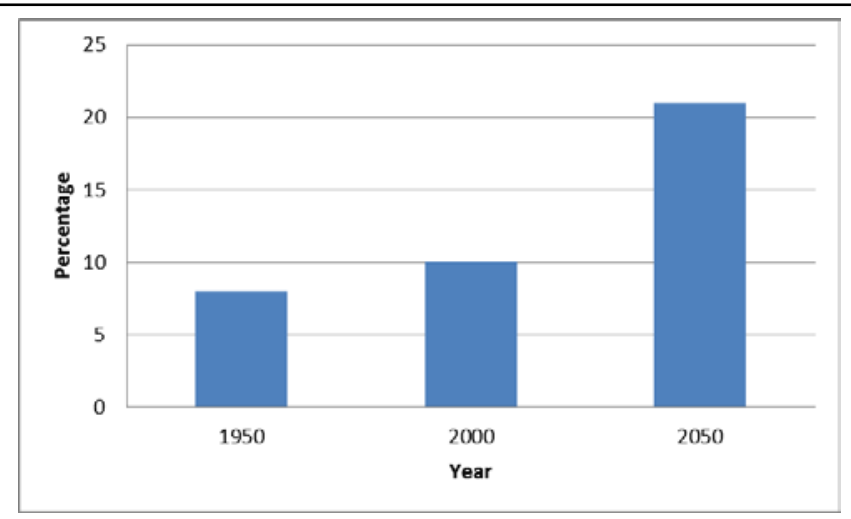

Fig. 1. Proportion of population older than 60 years old in 1950-2050 [6].
The population of aged 65 or above was estimated 524 million people (equivalent to 8\% of the world's population) in 2010 [6] This figure will be tripled (equivalent to $16 \%$ of the world's population) by 2050. Compared to adults below 65 years of age, elderly people have higher chances to suffer from illnesses and require more medical care. networks (WSNs) and associated medical devices become more readily available.

The shortage of medical students who graduate each year is a growing concern. Currently, at least 2 million medical personnel, including doctors and nurses, are needed. It is difficult to fill the vacancies of medical personnel in the coming few decades. The side effects of people living longer and lack of inadequate numbers of medical personnel/healthcare professionals seems to be insolvable. As a result, WSNs are believed to be the best way to tackle the healthcare issues because wireless communication allows people to roam anywhere and have ubiquitous access to network resources, documents and applications. Taking advantages of mobility, easy implementation and lack of cables, the market has shifted to tremendous growth in wireless devices.

WSNs are formed by extension of body sensor networks (BSNs). Sensors are used to measure biometrics, such as body temperature, electrocardiogram (ECG) signal, and blood pressure. The signals are collected and sent to a healthcare gateway, before being routed to a central system [7]. The BSN is responsible for a single user, whereas WSN handles numerous users and aggregate user's biometrics in a whole network. Networked computing [8] and experimental analysis [9] have been carried out for health monitoring using WSNs using Bluetooth and ZigBee. Besides health monitoring, autodiagnosis of diseases like cardiovascular diseases [10-12] and inflammation [13] have gained more concern. Machine learning algorithms can be used to train with patients' databases. Then, the ECG and wrist pulse signals measured by sensors keep testing by the trained algorithm. Once an outbreak of diseases is detected, an emergency call or a medical record will be made automatically. This reduces the work load of medical personnel and increases the probability of outcome.

WSNs in healthcare are limited to research laboratories and not yet practically adopted by hospitals and clinics. Current challenges include how to guarantee the accuracy of the captured biometrics. It is hoped this problem will be solved soon. The most important issues are security and coexistence with other medical equipment. The sensitive personal data of residents and patients must be protected and only allowed the access by authorized persons. This again comes to the same challenge in the previous sub-section. However, it is likely to be costly for a company to carry out a study on coexistence between medical equipment and the wireless technologies in WSNs. It is possible that medical institutions or governments should play a greater role in this.

Auto-diagnosis of diseases is a key feature for future in healthcare networks and more important than measuring the biometrics in [7]-[9]. A few open access databases can be utilized for training using machine learning algorithms. For cardiovascular diseases, there are more than 20 common types in general, whereas the largest database, PHYSIOBANK [14], 
has less than $50 \%$ completeness in terms of numbers of species and samples. Some data are missing and of small samples which are not sufficient for training. There are two major reasons why only a few hospitals take part in sharing the signals for diseases classification. Firstly, the signals are related to privacy of the recorder. Secondly, there is conflict of interest between the auto-diagnosis of diseases and medical personnel. Some are realized to lose their jobs if machines can replace their roles.

Aging and inadequate supply of medical personnel will force governments to increase the expenditure in healthcare. WHO has reported every year that the ratio of medical personnel to residents is always unacceptable and no policy has been formulated [15]. Therefore, the conflict of interests between medical personnel and WSNs in health cannot be assumed. Based on the concern in security, privacy and safety, studies will be started from individual houses to housing estate. When the obstacles are solved, the applications will move into clinics and hospitals. In aspect of auto-diagnosis of diseases, the system will act as rapid test, and the final decision is still reserved by doctors. Cardiovascular diseases are prioritized as number one because it contributes to more than one-quarter of the annual global death, and of the leading cause of death [16]. It was estimated that 17.3 million people died from cardiovascular diseases in 2008 and the figure will be increased to 22.3 million by 2030 [16], [17]. ECG signals have been applied in diagnosis of cardiovascular diseases for about one century [18]. Evidences have proved the accuracy and reliability using ECG signals. Hospitals around the world will share the datasets of ECG signals of different types of cardiovascular diseases. Possibly, the data collection for ECG datacase can be carried out according to the following five categories, rheumatic heart disease, hypertensive heart disease, ischemic heart disease, cerebrovascular disease, and inflammatory heart disease, and then divided into subcategories, in which there are different types of diseases in each subcategory. Machine learning algorithms can effectively extract the features from ECG signals and resulting from the classifier for auto-diagnosis. After that, other kinds of diseases can imitate the same means, as cardiovascular diseases.

\section{B. Smart Grid}

A Smart City is a mega project that connects all devices, citizens, applications and services together to render a sustainable city development as well as improve the quality of life for citizens. The annual outcome of energy related management is about 50 US billion dollars [19] and Advanced Metering Infrastructure (AMI) is an important milestone of energy management. AMI is one of the hottest research and commercial applications which aims at providing an infrastructure for two-way communication between smart meters of end users and utilities. In AMI, smart meters will be installed in every apartment or office at the end user site; the smart meters continually collect the electricity consumption profile and return this to the server at every pre-defined interval. Generally, a display unit is provided for end users to allow browsing the hourly, daily, monthly, and yearly electricity consumption. This is intended to change behavior and achieve a bidirectional effort to reduce the electricity wastage by utilities and customers.

Such mega-scale connection is one of the major obstacles for realizing a Smart Grid. Therefore, large scale WSN may be the key development for smart grid. There are already potential solutions for large scale AMI. In [20-22], high-traffic communication in BANs with high rises is proposed. A multiinterface management framework was defined and designed to coordinate the operation between multiple interfaces based on a tree-based mesh (T-Mesh) ZigBee topology, which supports both mesh and tree routing in a single network [20]. Based on [20], interference mitigation and optimization on the network was further developed afterwards for high rises [22]. Channel swapping algorithm was designed for ZigBee based AMI and optimal solutions by considering BER delay and transmission rate was obtained.

However, the aforementioned AMIs [22] have not addressed the power consumption issue. Since in reality portability is demanded, a low power solution is desperately required. In addition, the data centralization at the building coordinator is massive and such large data size requires extra costs in terms of bandwidth, latency and price. It is much more efficient if the meter reading of each floor can be sent out to the base station directly without data centralization. This requires large-scale and wide-area wireless network, which requires low power wide area network (LPWAN) technologies. For example, Narrowband IoT (NB-IoT), being developed in the 3rd generation partnership project (3GPP), promises to provide improved coverage $(1.5 \mathrm{~km})$ for massive number (around 100,000) of low-cost devices with low device power consumption (battery life more than 10 years) in delay-tolerant applications [23].

Alongside, AMI and NB-IoT, smart antenna draws attention to wireless communication, which may also be a potential facility for future AMI design. Smart antennas are techniques that use beamforming algorithms to steer the main beam of antenna array toward desired signal and create null points to sources of interference. For narrowband signals, the simplest weighted signal selection is the one that enhances the desired signal by aligning the phase shift in all sensors, and then adding the weighted signals to find the beamformer output [24]. Some processing methods use the statistical correlation of the data to design the weights. [25] These methods adaptively reduce the interference signal levels to that of the isotropic noise, which is the same as to maximize the output signal-to-interference-plus-noise ratio (SINR). These beamformers are referred to as the optimum fully adaptive beamformers (FAB) which fully adaptive is given because the beamformers use adaptation on all sensor weights [26]. However, the computational complexity of these narrowband beamforming is extremely high when the user number is large. Therefore, low computational complexity narrowband beamforming should be developed for massive users in AMI development.

However, MIMO antenna can enhance the SNR in AMI network. It is expected that the overall link budget will be better with MIMO design. Link budget can reflect the communication quality at the receiver. Especially if the uplink 
and the downlink frequencies are similar, the calculated signalto-noise ratios (SNRs) at transmitters and receivers using link budget equation can reveal whether two channels are corrupted or not. The empirical formula of link budget equation is [22]:

$$
P_{R X}=P_{T X}+G_{T X}+G_{R X}-L_{T X}-L_{R X}-L_{P}
$$

where $P_{R X}$ is the received power, $P_{T X}$ is the transmitter output power, $G_{T X}$ and $G_{R X}$ are MIMO-based transmitter gain and receiver gain, $L_{T X}$ and $L_{R X}$ are transmitter loss and receiver loss including insertion loss and back-off loss etc, and $L_{p}$ is the path loss.

\section{Localization}

Most countries are increasingly working on smart city projects. Smart logistics and transportation is one of the key applications under the smart city initiatives. Localization appears to be an important technological element in the smart logistics and transportation development. For example, supply chain management has been a dominant industry in Hong Kong for years and expanding with an enormous speed. Due to its unique special global location, Hong Kong has become the transportation hub connecting Mainland China with the whole world. At the same time, supply chain management is a sophisticated and mature technique that requires simultaneous monitoring from many aspects. Parallel transportation management systems have been used for ten years [27], enabling customers to track the location of package locally after ordering. With the uprising of TaoBao and TianMao, the requirements of reliable logistics are significantly increased. According to the China Satellite Navigation Office, experimental results show that the combination of the BeiDou and GPS bring a more accurate location tracking (10 meters error) which would be a great advantage in delivering packages at long distances, say, from Beijing to Hong Kong. Customers would know the location of packages once they placed an order. Within a city, local logistics and supply chain companies can utilize RFID tags to identify the lading or destination. Furthermore, by introducing Cloud computing technology, business parties or individuals are able to access the intelligent traffic clouds to get what they want or share what they know. From business view, they are continuously updating their latest information to potential customers as well as customer services. Customers can check whether the products are available or where the packages are located. Machine to machine (M2M) is another uprising technologies which has been used in healthcare and home management [28-29].

Therefore, an interoperable localization system would seem to be a logical direction for future logistic development. Locations of trucks would be continuously monitored by a layer where raw locations and other are further data are analyzed and then sent to cloud computing servers for distributions to manufacturers or customers. The Cloud would also act as a platform for displaying companies latest products or provision of services to customers. For illustration, the following diagram outlines the whole system which is a private Cloud implementation.
Moreover, in future developments, Inter-Cloud would be much efficient in distributing information or seeking potential customers in logistic aspect. The idea of Inter-Cloud is that since single cloud does not have unlimited physical resources and hence sharing among clouds over distances is probably a final trend of Cloud services. The importance of Inter-Cloud technique aims at building a network for sharing their information. This is mainly due to the fact that warehouses are distributed within a large region.

The received signal strength (RSS), $P_{R}$, reveals the communication quality and is one of the typical indicators for localization. To provide a wider area of coverage for localization, handover in WSNs is needed. The handover strategy will affect the data storage efficiency, the computation cost and the quality of data transmission. It is noted that the received signal strength will decay with an increase of the propagation distance. The decay is normally reflected in the path loss exponent $\alpha$ (refer to (2)) which varies with the propagation medium and should be determined experimentally. The empirical formula of the path loss model can be expressed as:

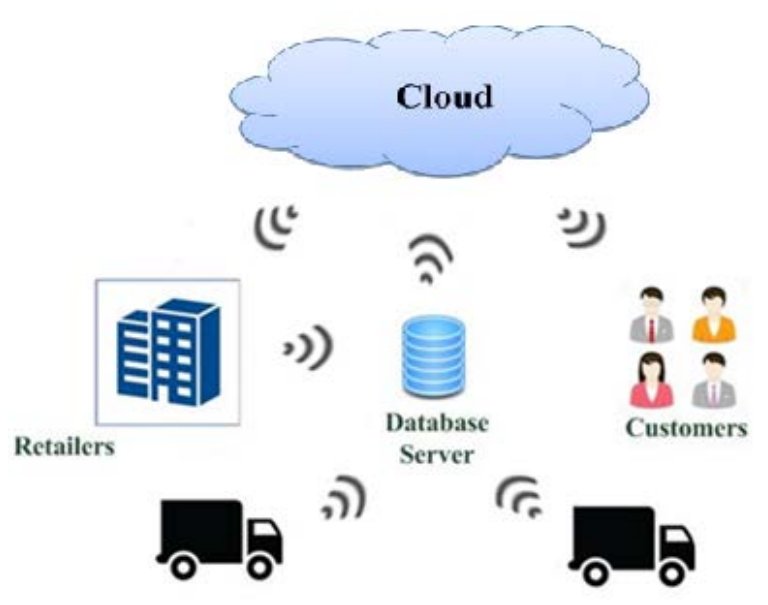

Fig. 2. System overview in cloud network localization.

$$
P_{R}=P_{0}\left(\mathrm{~d}_{0}\right)_{\mathrm{dB}}-10 \alpha \log _{10} \frac{\mathrm{d}}{\mathrm{d}_{0}}+v_{\sigma}
$$

where $P_{R}$ is the calculated received signal strength (RSS) using empirical formula $(2), P_{0}\left(d_{0}\right)$ is the reference RSS at reference distance $d_{0}, d$ is the transmission distance, and $v_{\sigma}$ is the noise figure with deviation $\sigma$.

By measuring $P_{R}$ at a distance $d$ and also the reference RSS at reference distance $d_{0}, \alpha$ can be calculated. Typically, the values of $\alpha$ of indoor environment and outdoor environment are very different. Therefore, a good estimation of $\alpha$ and the link budget-related parameters will help to establish a good communication environment.

\section{Wireless Networks and Denial of Service Attacks}

With the advent of widespread use of wireless networks there is always the potential for direct unauthorized connection 
or denial of service attacks. Unauthorized intrusion can usually be mitigated by tightly controlled security protocols provided there are no 'back doors' left open unintentionally or intentionally. However, denial of service attacks can be initiated by saturated by users who have legitimate access to the network - such attacks are usually detected long after the event and when forensic evidence has long since been discarded. Wireless networks all rely on RF communications (except for direct line of sight optical or fibre based solutions). It is easy to generate an RF signal corresponding to the frequency of the wireless network to be attacked with a signal which is much greater in signal strength that the devices that usually connect to the network. This causes an immediate local denial of service - these are usually easily detected because of the local impact. However, the only way to find the source of interference is through very careful direction finding. Unauthorized intrusion arguably represents the greatest longterm threat because the intruders have legitimate access to the network.

Another threat to wireless network reliability is the available RF bandwidth - there are only a finite number of channels that can be supported in a particular region/area. Whether these are highly regulated in terms of allowable bandwidth is a question for the authorities.

As an aside, the link between future vehicles and infrastructure might only exist for a short time. The IEEE 802.11p allows exchange data without the need to establish a basic service set and without the need to wait for authentication before exchanging data. For that reason, because such nodes are not authenticated, the authentication and data confidentiality is high risk.

\section{CONCLUSION}

Wireless Networks are increasingly taking advantage of the opportunities offered by wireless technologies to serve new and original smart city functions and solutions. Certainly, the scalability, robustness and flexibility of the smart city networks need to be addressed. In this paper, an overview of the state-of-the-art development on smart city wireless networks has been given with the snapshot on typical wireless technologies. Visions and opportunities of key applications including healthcare, smart grid and localization have been discussed. It is believed that wireless technologies in smart city development will draw more and more attractions in both commercial and research domains. However, failure to manage the security and use of the bandwidth used for wireless networks will lead to completely inoperable systems and services that rely on their operational availability. There is no such thing as unlimited bandwidth so care must be taken to use it wisely.

\section{REFERENCES}

[1] K. David, S. Dixit, and N. Jefferies, “2020 vision,” IEEE Veh. Technol., vol. 5, no. 3, pp. 22-29, Sep. 2010.
[2] G. Patti, L. Leonardi and L. L. Bello, “A Bluetooth Low Energy realtime protocol for industrial wireless mesh networks," IECON 2016 42nd Annual Conference of the IEEE Industrial Electronics Society, Florence, 2016, pp. 4627-4632.

[3] Bluetooth Specification, Mesh Profile v1.0, Bluetooth SIG - Mesh Working Group, July 2017.

[4] LoRaWAN TM 101 - A Technical Introduction, 2016.

[5] "IEEE 1609 - Family of Standards for Wireless Access in Vehicular Environments (WAVE)," U.S. Department of Transportation, Nov. 2014.

[6] Global Health and Aging, World Health Organization, 2011.

[7] S. -L. Chen, H. -Y. Lee, C. -A. Chen, H. -Y. Huang, and C. -H. Luo, "Wireless body sensor network with adaptive low-power design for biometrics and healthcare applications,” IEEE Syst. J., vol. 3, no. 4, pp. 398-409, Dec. 2009.

[8] A. Jindal, and M. Liu, "Networked computing in wireless sensor networks for structural health monitoring," IEEE/ACM Trans. Netw., vol. 20, no. 4, pp. 1203-1216, Aug. 2012.

[9] H. Yan, H. Huo, and M. Gidlund, "Wireless sensor network based ehealth system - Implementation and experimental results,” IEEE Trans. Consum. Electron., vol. 56, no. 4, pp. 2288-2295, Nov. 2010.

[10] Z. Zhao, L. Yang, D. Chen, and Y. Luo, "A human ECG identification system based on ensemble empirical mode decomposition," Sensors, vol. 13, no. 5, pp. 6832-6864, May 2013.

[11] S. I. Safie, J. J. Soraghan, and L. Petropoulakis, "Electrocardiogram (ECG) biometric authentication using pulse active ratio (PAR),” IEEE Trans. Inf. Forensics Security, vol. 6, no. 4, pp. 1315-1322, Dec. 2011.

[12] B. Schumacher, S. Spehl, A. Langbein, A. Schade, S. Kerber, and M. Koller, "Regular tachycardia with broad QRS complex: Differential diagnosis on 12-lead ECG,” Herzschrittmacherther Elektrophysiol, vol. 20, no. 1, pp. 5-13, Apr. 2009.

[13] Y. Chen, L. Zhang, D. Zhang, and D. Zhang, “Computerized wrist pulse signal diagnosis using modified auto-regressive models,” J. Med. Syst., vol. 35, pp. 321-328, Sep. 2009.

[14] ECG Database, PhysioBank, Physionet.

[15] Global Atlas of the Health Workforce, World Health Organization, Geneva, 2012.

[16] M. Global status report on noncommunicable diseases 2010, World Health Organization, Geneva, 2011.

[17] C. D. Mathers, D. Loncar, "Projections of global mortality and burden of diseases from 2002 to 2030,” PLoS Med., vol. 3, no. 2, Nov. 2006.

[18] A.B. D. Luna, Clinical Electrocardiography: A Textbook. New York: Futura Publishing Company, 1998.

[19] F. Lienert, L. Platchkov, "Smart meter roll-out for the non-domestic sector (GB),” 2012.

[20] H. Y. Tung, K. F. Tsang, K. T. Chui, H. C. Tung, H. R. Chi, G. P. Hancke, K. F. Man, “The Generic Design of a High-Traffic Advanced Metering Infrastructure Using ZigBee”, IEEE Transactions on Industrial Informatics, vol. 10, pp. 836-844, Feb 2014.

[21] H. Y. Tung, K. F. Tsang, K. L. Lam, "ZigBee sensor network for Advanced Metering infrastructure”, Digest of Technical Papers International Conference on Consumer Electronics (ICCE), pp. 1-2, Sep 2010.

[22] H. R. Chi, K. F. Tsang, K. T. Chui, H. S. H. Chung, B. W. K. Ling, L. L. Lai, "Interference-Mitigated ZigBee Based Advanced Metering Infrastructure”, IEEE Transactions on Industrial Informatics, vol. 12, pp. 572-584, Apr 2016.

[23] 3GPP, “Revised work item: Narrowband IoT (NB-IoT),” RP-152284, December 2015.

[24] J. E. Piper, "Beamforming narrowband and broadband signals, sonar systems,” InTech, 2011.

[25] B. Rafaely, "Spatial Sampling and Beamforming for Spherical Microphone Arrays," 2008 Hands-Free Speech Communication and Microphone Arrays, Trento, 2008, pp. 5-8.

[26] A. M. Torres, J, Mateo and L. M. Vicente, "Performance analysis of narrowband beamforming using fully and partial adaptive beamformers 
with a spherical array,” Multidimensional Systems \& Signal Processing, Springer, 2016.

[27] A. M. Kuo, "Opportunities and challenges of cloud computing to improve health care services,” Journal of medical Internet research, vol. 13, no. 3, 2011.

[28] S. J. Jung, R. Myllylä and W. Y. Chung, "Wireless Machine-toMachine Healthcare Solution Using Android Mobile Devices in Global Networks," in IEEE Sensors Journal, vol. 13, no. 5, pp. 1419-1424, 2013.
[29] M. Chen, J. Wan, S. Gonzalez, X. Liao and V. C. M. Leung, "A Survey of Recent Developments in Home M2M Networks," in IEEE Communications Surveys \& Tutorials, vol. 16, no. 1, pp. 98-114, 2014.

[30] E. Toscano, L. Lo Bello, "Comparative assessments of IEEE 802.15 4/ZigBee and 6LoWPAN for low-power industrial WSNs in realistic scenarios", IEEE International Workshop on Factory Communication Systems (WFCS), Lemgo/Detmold, Germany, pp. 115-124, May 2012. 